\title{
Testing of Partial Discharges in Generator Coil Bars with the Help of Calibrated Acoustic Emission Method
}

\author{
F. Witos ${ }^{a, *}$, Z. GACEK ${ }^{b}$ AND Z. OPILSKI ${ }^{a}$ \\ ${ }^{a}$ Institute of Physics, ${ }^{b}$ Institute of Power Systems and Control \\ Silesian University of Technology \\ Krzywoustego 2, 44-100 Gliwice, Poland
}

\begin{abstract}
Investigation results of acoustic emission signals coming from partial discharges within chosen generator coil bar, in relation to measurement results of apparent charge introduced by partial discharges sources, are presented in the article. Analysis is based on amplitude distributions calculated for acoustic emission signals, ordered at different measuring points for different values of the supply voltage. In order to assign acoustic emission signal to its advance degree a descriptor named by an acronym ADP (defined by authors) is calculated for each amplitude distribution. Descriptor families describing acoustic emission signals measured at particular measuring points (for different supply voltages) are presented in relation to apparent charge introduced by acoustic emission sources. These families concerning acoustic emission signals, measured at different measuring points for identical supply voltage, enable us to locate acoustic emission sources with maximum activity.
\end{abstract}

PACS numbers: 43.40.Le, 43.60.Lg, 52.80.s, 77.22.Jp, 84.70.+p

\section{Tested object and testing description}

Several hundreds of generator coil bars is produced yearly in Poland. Each of these bars, before issuing of permit to work, is applied in qualification tests. One of these tests consists in determination of partial discharges (PD) level, carried out by means of electric method [1]. Order of magnitude of admissible apparent charge in the case of considered objects is nanocoulombs.

Generator coil bar is a very interesting test object because:

- it is a complex insulating system (electrodes, semiconductor areas to control electric field, complex insulating layers, conducting layer) where different PD sources typical of solid material may appear;

*corresponding author; e-mail: franciszek.witos@pols.pl 
- acoustic emission (AE) sensors can be installed at many points of a tested bar which creates possibilities for identification of AE signals and location of PD sources.

Generator coil bars are produced among other things by the firm ENERGOSERVICE S.A. in Lubliniec, Poland. Testing of these bars issuing of permit to work is carried out at several specialist stands.

Authors of the article proposed a prototype stand applied to investigate PD within generator coil bars, composed of original AE measuring system DEMACOMP $[2,3]$ and professional computer measuring system TE $571[4-6]$.

Within a framework of preparation for testing with the help of electric method TE 571 system was calibrated by means of a model apparent charge and it was prepared to automatic measurements of the supply voltage. In this way interferences coming from additional connections with measuring apparatus have been eliminated.

It has been established that testing carried out by AE method concerns only a slot part of the bar. This area is limited by additional earthing electrodes; between them there are six evenly distributed measuring points P1, P2,., P6 (see Fig. 1).

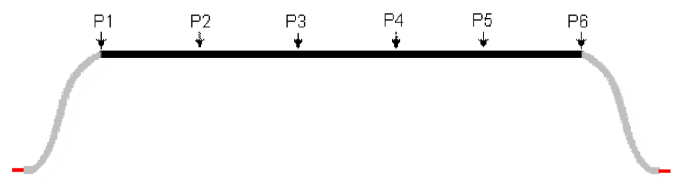

Fig. 1. Arrangement of measuring points in chosen generator coil bar during testing of PD with the help of AE method.

AE sensors (being elements of K1, K2 and K3 measuring lines) have been placed at measuring points P1, P2 and P3. Each tested bar was energized by the supply voltage whose values were selected from the range of $\left(0,2 U_{N}\right)$, measurements have been carried out using two methods simultaneously. During measurements made by means of electric method every PD signal was detected by $120 \mathrm{~s}$ for each value of applied supply voltage (time period of data registration demanded to realize calculations made in analysis mode and by means of TEAS program). $\mathrm{AE}$ signals were recorded for each value of selected supply voltages within time periods of $2 \mathrm{~s}$. After testing for all selected values of the supply voltage AE sensors have been assembled in next position: (P2, P3, P4), (P3, P4, P5)... Registration of $\mathrm{AE}$ impulses were made for the next position of AE sensors. This process has been repeated until measuring data from all $\mathrm{AE}$ sensors (in all measuring lines) was obtained.

The purpose of testing was analysis of PD phenomena occurring within generator coil bars with the help of the so-called calibrated acoustic emission (CAE) method. According to a such method, measurements are carried out by means of 
AE method and - simultaneously - by means of electric method (it concerns apparent charge). Such an approach enables us to analyze PD phenomena occurring within generator coil bars basing on $\mathrm{AE}$ method and then to connect obtained results with apparent charge introduced by active PD sources.

Below, there are testing results together with an analysis for a chosen "D" bar (coil bar of the generator $120 \mathrm{MW}, U_{N}=13.8 \mathrm{kV}$ ). The bar is interesting to analysis of PD sources whose apparent charge values are on the level of several $\mathrm{nC}$ (nanocoulombs); apparent charge value introduced by PD sources decreases when the supply voltage grows bigger. Measuring results of apparent charge introduced by PD sources are presented in Table (tested PD band includes a range between $40 \mathrm{~Hz}$ and $400 \mathrm{kHz})$.

TABLE

Measuring results of apparent charge within "D" bar $\left(U_{N}=13.8 \mathrm{kV}\right)$.

\begin{tabular}{c|c|c|c|c|c}
\hline \hline$U[\mathrm{kV}]$ & 10 & 15 & 20 & 25 & 30 \\
$Q_{p}[\mathrm{nC}]$ & 3.5 & 3.8 & 2.2 & 2.2 & 2.0
\end{tabular}

\section{Determination of dominant bands in AE signals coming from PD within generator coil bars}

At the beginning, PD occurring within generator coil bars were tested using AE sensors of R6 and WD type because summary of transfer band for two types of
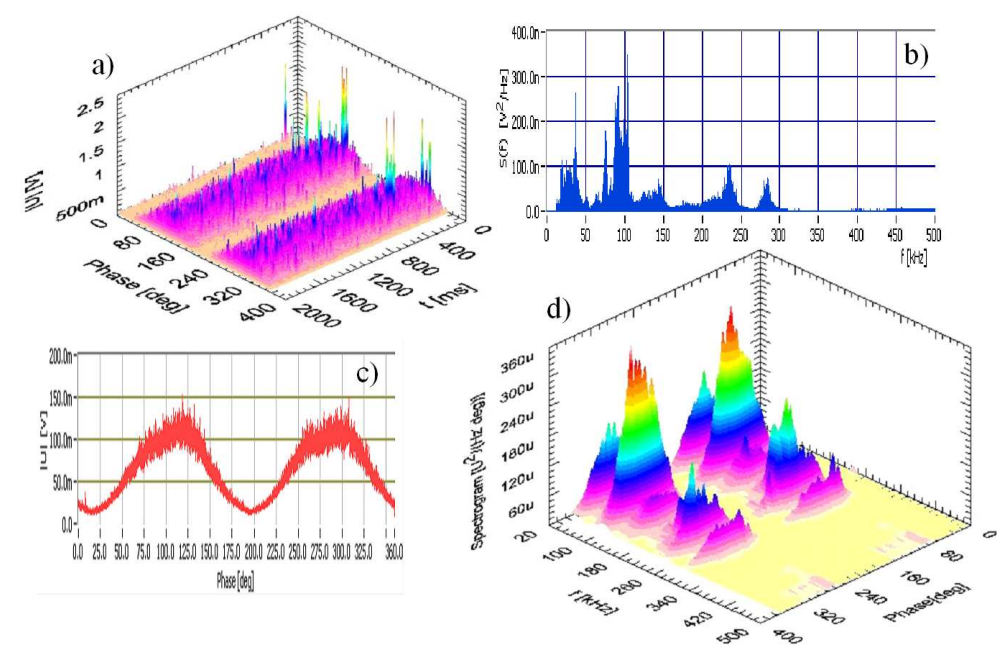

Fig. 2. Description of AE signal ordered in chosen measuring conditions ("D" bar, $30 \mathrm{kV}, 2 \mathrm{nC}$, measuring channel $\mathrm{K} 3(20 \mathrm{~dB}$, WD), measuring point P1): (a) phase-time characteristic, (b) averaging phase characteristic, (c) frequency characteristic, (d) averaging STFT spectrogram. 
sensors covers transfer band of the measuring system DEMA-COMP $(20-500 \mathrm{kHz})$. Exemplary description of AE signal recorded within "D" bar and measuring line with WD sensors is presented in Fig. 2. All elements of this description are obtained using filtration of AE signal made by means of band-pass filter of 5 order with a band of 20-500 kHz. In diagrams (a) and (d) in Fig. 2 there are the following bands in the spectrum of AE signal: $20-40 \mathrm{kHz}, 80-110 \mathrm{kHz}, 120-150 \mathrm{kHz}$, 220-240 kHz and 270-290 kHz. Appearance of such high frequencies in AE signals is confirmed by other publications [7-10]. Frequency characteristic of measuring line for frequencies greater than $150 \mathrm{kHz}$ is flat so that analysis of $\mathrm{AE}$ properties has been carried out in the band of $150-500 \mathrm{kHz}$.

\section{Basic properties of AE signals coming from $\mathrm{PD}$ within generator coil bars}

Preliminary description of an exemplary AE signal coming from PD, generated within generator coil bar, is presented in Fig. 3. Characteristics (d), (e) and
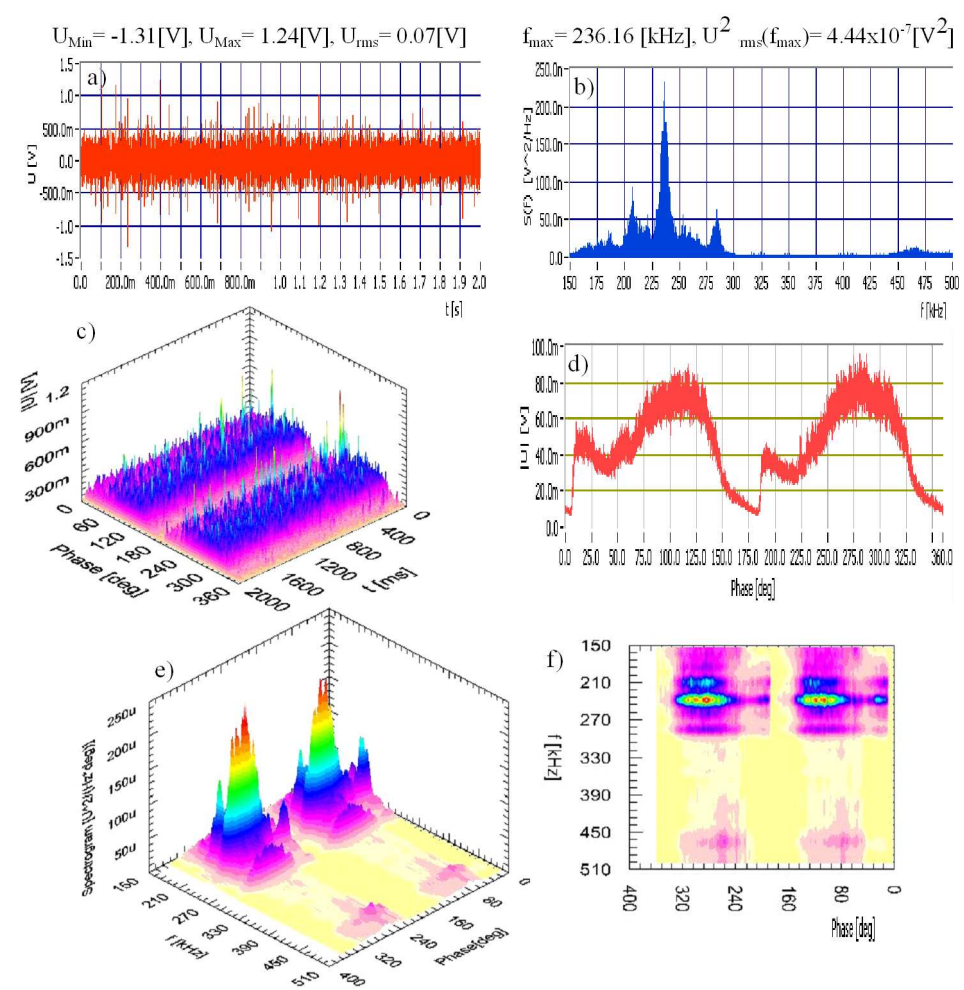

Fig. 3. Preliminary description of AE impulses: (a) AE signal, (b) frequency characteristic, (c) phase-time characteristic, (d) averaging phase characteristic, (e) and (f) averaging STFT; measuring conditions: "D" bar, $30.3 \mathrm{kV}, 2.0 \mathrm{nC}, \mathrm{K} 3$ measuring line $(20 \mathrm{~dB}, \mathrm{WD})$, measuring position P2. (a), (c), (e) $U_{\min }=-1.19 \mathrm{~V}, U_{\max }=1.29 \mathrm{~V}$, $U_{\mathrm{rms}}=0.07 \mathrm{~V}$. (b), (d), (f) $f_{\max }=236.16 \mathrm{kHz}, U_{\mathrm{rms}}^{2}\left(f_{\max }\right)=4.44 \times 10^{-7} \mathrm{~V}^{2}$. 
(f) represent suitable quantities, averaging in all periods of the supply voltage. Every characteristics show periodic character of PD, revealed by appearance of maximum of tested quantity - twice during supply voltage period. Characteristic (c) in Fig. 3, i.e. phase-time diagram, is useful to analyze AE signal amplitude for a selected phase during particular periods of the supply voltage. It reveals two features: greater values of a signal amplitude occur in two phase intervals (two "corridors" in a diagram) whereas - for next supply voltage periods - phase diagrams of amplitude signals reveal fluctuation of values. First property is connected with periodical character of PD whereas the second testifies the random character of tested phenomenon.

Description of noises in a measuring line is presented in Fig. 4. AE signal and noise in a measuring line, ordered during two successive periods of the supply voltage, is presented in Fig. 5.
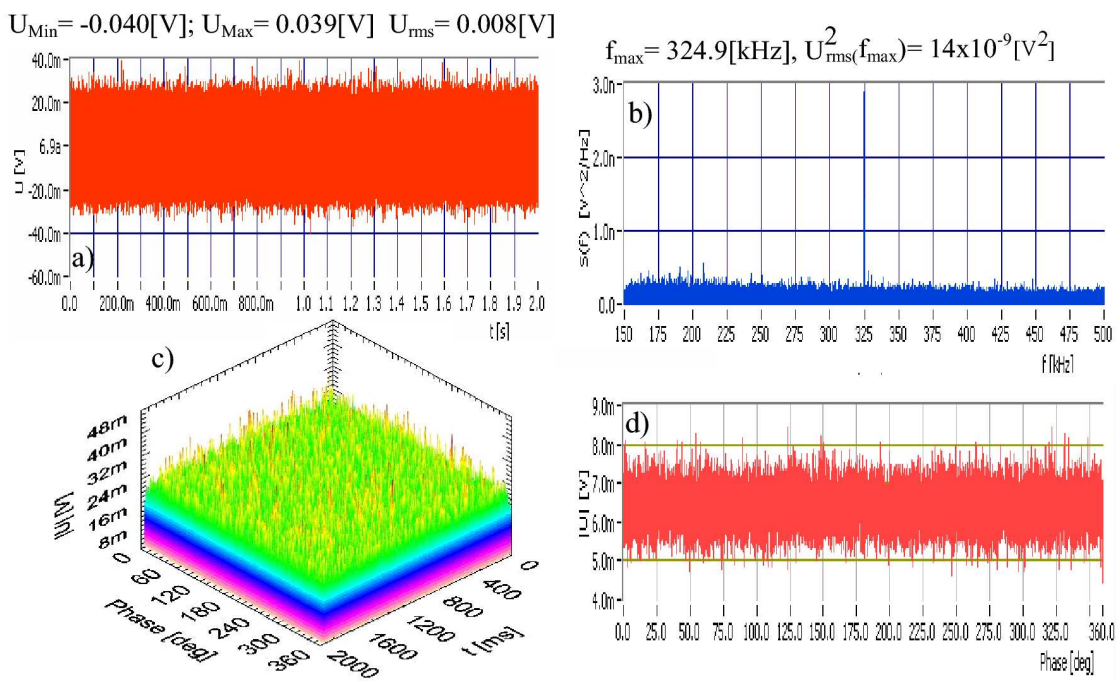

Fig. 4. The noise within a measuring line: (a) impulse, (b) frequency characteristic, (c) phase-time characteristic, (d) averaging phase characteristic; measuring conditions: "D" bar, $0 \mathrm{kV}, \mathrm{K} 3$ measuring line $(20 \mathrm{~dB}, \mathrm{WD})$, measuring position P2. (a), (c) $U_{\min }=-0.040 \mathrm{~V}, U_{\max }=0.039 \mathrm{~V}, U_{\mathrm{rms}}=0.008 \mathrm{~V}$. (b), (d) $f_{\max }=324.9 \mathrm{kHz}$, $U_{\mathrm{rms}}^{2}\left(f_{\max }\right)=1.4 \times 10^{-8} \mathrm{~V}^{2}$.

Detailed analysis of preliminary descriptions of AE signals coming from PD within generator coil bar, detected at different measuring points for different values of the supply voltage, reveals changes of $\mathrm{AE}$ signal properties depending on value of the supply voltage and position of a measuring point. Appearance of these changes shows that proposed method of testing enables us to analyze deformation processes taking place within the object. 

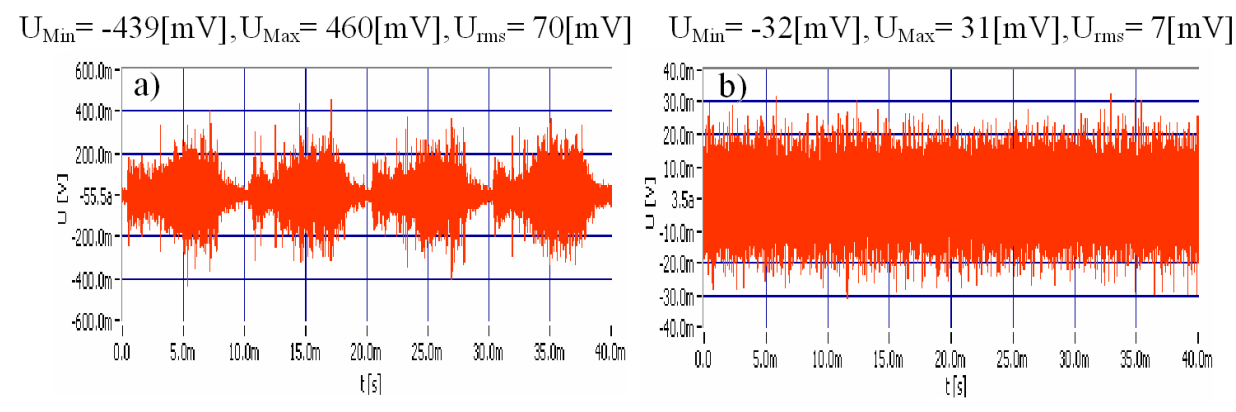

Fig. 5. Initial fragment of detected signals during two periods of the supply voltage: (a) from Fig. 3a $\left(U_{\min }=-439 \mathrm{mV}, U_{\max }=460 \mathrm{mV}, U_{\mathrm{rms}}=70 \mathrm{mV}\right)$, (b) from Fig. $4 \mathrm{a}$ $\left(U_{\min }=-32 \mathrm{mV}, U_{\max }=31 \mathrm{mV}, U_{\mathrm{rms}}=7 \mathrm{mV}\right)$.

\section{Amplitude distributions of $\mathrm{AE}$ signals as an information source on deformation processes during PD within generator coil bars}

Detailed analysis of AE signal properties within generator coil bars has been carried out on the base of amplitude distributions of AE signals. In order to make such an analysis, special procedures have been worked by authors of the article, relating to: calculation of amplitude distributions of $\mathrm{AE}$ signals (using amplitude distributions of power of $\mathrm{AE}$ signal and its derivative with respect to discrimination threshold) as well as analysis of properties of amplitude distributions by means of descriptors.

In the present article the so-called ADP descriptor [11] is used. It is noted by the acronym amplitude distribution of power (of AE signal), defined as follows. A fragment of calculated amplitude distribution is selected. This fragment should correspond to discrimination threshold range $\left(U_{\mathrm{d}}, U_{\mathrm{g}}\right)$ where $U_{\mathrm{d}}$ is given by a minimum of amplitude distribution derivative, calculated with respect to discrimination threshold, whereas $U_{\mathrm{g}}$ is $90 \%$ of the maximum value of ordered $\mathrm{AE}$ signal. Such a fragment of a curve is approximated by a straight line; defined descriptor value is equal to the slope of this straight line. Described idea concerning calculation of ADP descriptor value is presented in Fig. 6.

Descriptor defined in such a way takes into account physical features of investigated phenomenon, connected with propagation and thickness of interconnected layer (logarithmic scale of values on amplitude distributions). It is not based on values measured directly (amplitudes, energies of signals etc.) but describes recorded AE signals, giving them the so-called advance degree. Descriptors are negative values: greater descriptor value (more plate fragment of a curve) marks higher (more) advanced degree of AE signal. Such a degree is connected with advance degree of a deformation process and takes into account regularity that this process takes place within an EA source, whereas EA signal is recorded at a measuring point. 

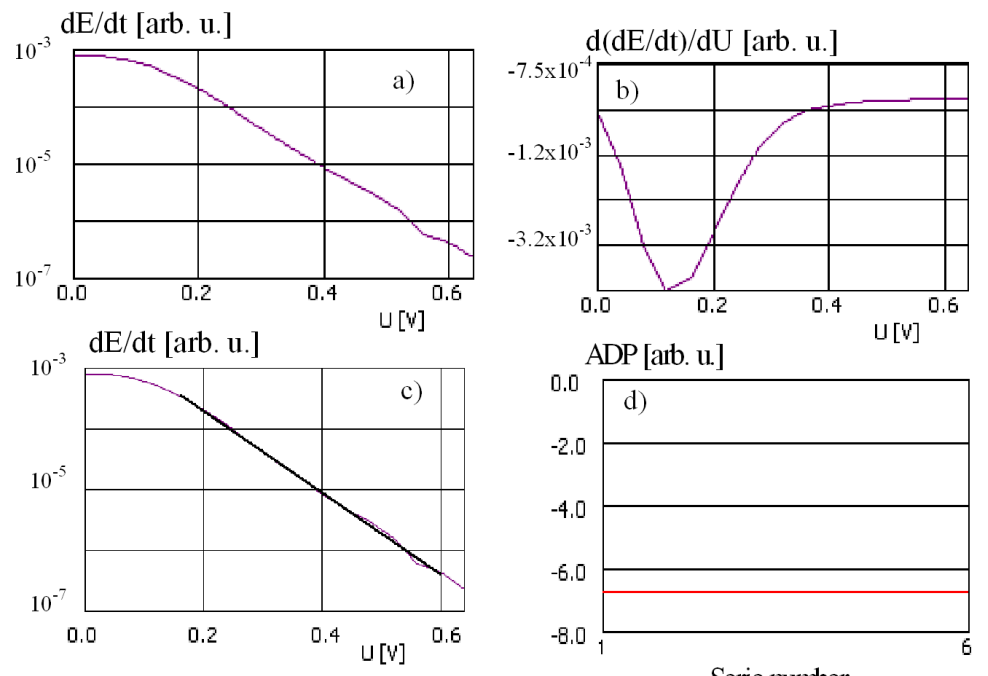

Serie number

Fig. 6. Calculation of ADP descriptor: (a) amplitude distribution of power of AE signal (from Fig. 4), (b) derivative of amplitude distribution of power of AE signal, (c) amplitude distribution with approximation Curie and threshold range $\left(U_{\mathrm{d}}, U_{\mathrm{d}}\right)$ marked by $\leftrightarrow$, (d) ADP descriptor resulting from the diagram in Fig. 6a.

Now, let us look at results of complete qualitative analysis of $\mathrm{AE}$ signals for their amplitude distributions, recorded at all measuring points of "D" bar (after filtration in the band of $150-500 \mathrm{kHz}$ ).

\section{Results of complete qualitative analysis of properties of amplitude distributions}

Complete qualitative analysis concerns amplitude distributions carried out for a group of $\mathrm{AE}$ signals, recorded at all six measuring points of tested bar. There are 24 signals analyzed at each measuring point (4 signals recorded for the following supply voltage: $0,19,15,20,25,30 \mathrm{kV}$ ). On the whole, it gave a group of $144 \mathrm{AE}$ signals recorded at measuring points P1-P6. Signals were filtrated in the band of $150-500 \mathrm{kHz}$ and - not before filtration - amplitude distribution of AE signals have been calculated. The following mean values have been calculated for amplitude distributions recorded at a given measuring point and for one value of the supply voltage: average of ADP descriptor and average standard derivation of this descriptor. Results are presented in Fig. 7.

Analysis of values and shapes of particular curves presented in Fig. 7 gives the following results:

- the least advanced deformation processes take place in the vicinity of the measuring point $\mathrm{P} 4$ (the curve increases monotonically and it is identified by authors as a "simple shape"; 


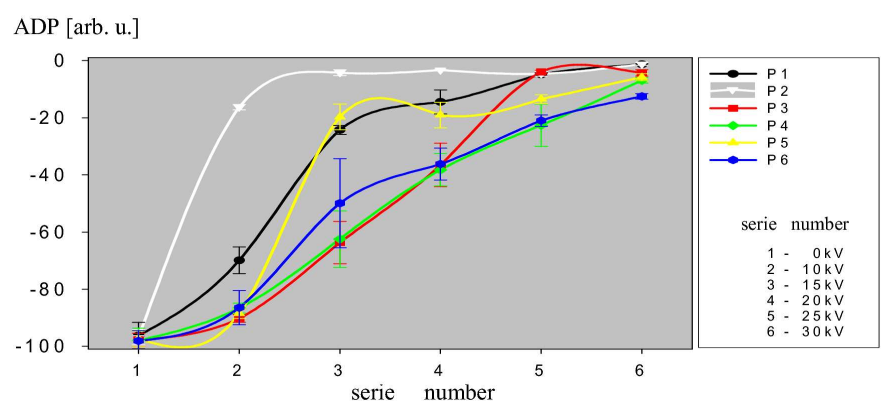

Fig. 7. The group of ADP descriptors for AE signal family ordered within all measuring points (for signal ordered at measuring points P1-P6 versus supplied voltages, "D" bar, in the band of $150-500 \mathrm{kHz})$.

- the most advanced deformation processes take place in the vicinity of the measuring point P2 (the curve does not increase monotonically and it is identified by authors as a "complex shape");

- ADP curve for the point P\% can be treated as "complex shape" one;

- ADP curves for points P1, P3 and P6 have "intermediate shape";

- signals recorded at the measuring point P2 are the most stable, whereas the great fluctuations are observed at measuring points $\mathrm{P} 6$ and $\mathrm{P} 4$;

- decisive influence on a global AE signal comes from PD sources situated in the vicinity of the measuring point P2.

\section{Location of PD sources with maximum activity in the band of $150-500 \mathrm{kHz}$}

ADP descriptors calculated for amplitude distributions can be divided into groups for the sake of signals recorded under similar supply voltages. Such a list is presented in Fig. 8; it is a base for location of PD sources with maximum activity $[7,11]$. According to this method - proposed by authors - location is carried out for particular supply voltages and consists in determination of the measuring point where a local maximum in the curve is observed.

Results of location of PD sources with maximum activity for "D" bar are as follows:

a) when the supply voltage is $10 \mathrm{kV}$, then the PD source with maximum activity is situated in the vicinity of the measuring point P2,

b) when supply voltages are $15 \mathrm{kV}$ and $20 \mathrm{kV}$, then the PD source with maximum activity situated in the vicinity of the measuring point P2 is still active but another source appears nearby the measuring point P5,

c) when supply voltages are $25 \mathrm{kV}$ and $30 \mathrm{kV}$, then local maxima remain identical values (as for $15 \mathrm{kV}$ and $20 \mathrm{kV}$ ) but differences between local maximum 


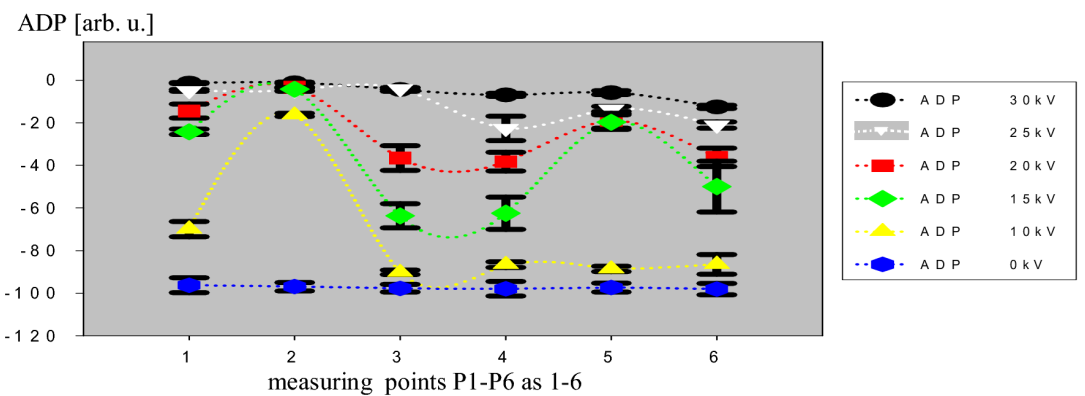

Fig. 8. The group of AE descriptors for AE signals ordered by WD sensor at measuring points $\mathrm{P} 1-\mathrm{P} 6$, "D" bar, in the band of $150-500 \mathrm{kHz}$ for different values of the supply voltage (maximum within each group locates the PD source with maximum activity).

and local minimum values in the curve are much smaller (comparing with curves for supply voltages $15 \mathrm{kV}$ and $20 \mathrm{kV}$ ).

Statement of descriptor families for particular supply voltages gives additional information about location of PD sources. AE signals for lower supply voltages are recorded only at measuring points situated nearby PD sources whereas $\mathrm{AE}$ signals for high supply voltages are recorded at all measuring points. In the last case, "weighing" of AE signals enables still to locate PD sources. Elongation of propagation path of AE signals when the supply voltage increases is caused by that order of magnitude of power of AE signals recorded under $25 \mathrm{kV}$ and $30 \mathrm{kV}$ is several range higher than power of AE signals recorded under $10 \mathrm{kV}$. Greater amplitude of AE signal enables us to detect AE signals at measuring points situated further from PD sources.

It is worth noticing that descriptors for repeatedly measured $\mathrm{AE}$ signals at different measuring points, being noises of a measuring path, are similar values: about 100 contractual units.

\section{Recapitulation}

Testing of partial discharges occurring in chosen generator coil bar have been made with the help of calibrated acoustic method which consists in simultaneous measurements of apparent charge (electric method) and AE signals (acoustic method).

Electric method shows that partial discharges appear in tested bar under the supply voltage of $7.5 \mathrm{kV}$. Qualitative measurements started from the supply voltage of $10 \mathrm{kV}$. Apparent charge reaches its maximum $3.8 \mathrm{nC}$ under the supply voltage of $15 \mathrm{kV}$. Subsequent increase in the supply voltage results in monotonic reduction of apparent charge, introduced by active PD sources: it attains $2.0 \mathrm{nC}$ under the supply voltage of $30 \mathrm{kV}$.

Analysis of particular signals is based on amplitude distributions and connected with them ADP descriptors. Such an analysis, including properties of ADP 
descriptor families, enabled us to locate two PD sources with maximum activity, situated in the vicinity of measuring points P2 and P5. ADP curves for these points do not increase monotonically for supply voltages over $15 \mathrm{kV}$. ADP curves at other measuring points are character ascending monotonically when the supply voltage increases. Significant contribution in global signal coming from PD sources gives the source situated nearby the measuring point P2.

\section{References}

[1] P. Bartnikas, IEEE Trans. Diel. Electr. Insul. 9, 763 (2002).

[2] F. Witos, Investigation of partial discharges by means of acoustic emission method and electric method, Wydawnictwo Politechniki Śląskiej, Gliwice 2008 (in Polish).

[3] T. Boczar, S. Borucki, A. Cichon, M. Lorenc, Mol. Quant. Acoust. 26, 35 (2005).

[4] A. Bejger, Z. Ranachowski, Mol. Quant. Acoust. 27, 227 (2006).

[5] F. Witos, Z. Gacek, Europ. Phys. J. Special Topics 154, 239 (2008).

[6] F. Witos, Z. Gacek, J. Phys. IV (France) 129, 173 (2005).

[7] Z. Gacek, P. Paduch, F. Witos, Mol. Quant. Acoust. 28, 271 (2007).

[8] T. Kaneko, A. Takemura, O. Takenouchi, Y. Sung, M. Otsuba, C. Honda, K. Tanaka, in: ISH - XIIIth Int. Symp. on High Voltage Engineering, Netherlands 2003, Ed. J.J. Smit, Milpress, Rotterdam 2003.

[9] E. Nazarova, A. Tcheryatian, Mol. Quant. Acoust. 28, 259 (2007).

[10] T. Boczar, S. Borucki, A. Cichon, Mol. Quant. Acoust. 28, 13 (2007).

[11] F. Witos, Z. Gacek, A. Opilski, Arch. Acoust. 27, 65 (2002). 